\title{
Computer modelling of the behaviour of actin gels
}

\section{from Dennis Bray}

UNDERSTANDING certain kinds of cell movements may require the use of a computer, to judge from two recently published papers by theoretical biologists G.M. Odell ${ }^{1}$ and G.F. Oster ${ }^{2}$. This applies especially to the slow amoeboid movements of animal cells as they crawl over surfaces, engulf large particles or pinch into two in the course of cell division, movements which all depend on complex physicochemical transformations in the amorphous protein gel just beneath the cell membrane.

While the biochemistry of the gel is fairly well understood (it contains actin, myosin and a medley of other proteins able to cross-link, fragment, cap or nucleate actin filaments-see ref. 3), its behaviour as a whole is difficult to predict. Most of the proteins are regulated by calcium ions, so that a transient wave of calcium through the cytoplasm is liable to be followed by solation, gelation, polymerisation and contraction. Moreover, the kinetics of these various effects are not the same, and transformations in one region of the gel have a mechanical and chemical influence on other regions. Seen from this perspective, even the simple extension of the leading edge of a fibroblast over a surface becomes a formidable skein of cause and effect.
Recognizing that only the language of mathematics is adequate to represent such interacting phenomena, Oster and Odell have been building detailed quantifiable models of actin gel behaviour. Their approach is to use a series of equations (also presented as word-pictures for nonmathematical readers) that represent the relationships between the stress, strain and viscosity of actin networks, and biochemical parameters such as calcium ion concentration. Combined into a multidimensional model, these provide an impressively accurate, and at times counter-intuitive, view of gel behaviour.

In the first paper that illustrates the genre, Odell presents a detailed descriptive model of the rapid repetitive shuttling of cytoplasm in the acellular slime mould Physarum $^{1}$. A series of biochemically plausible and experimentally verifiable equations describing the properties of an actin gel lead to the accompanying illustration of its behaviour. Here, the stretch imposed on a small volume of cytogel is related to the force it exerts and to the concentration of a diffusible regulatory component such as calcium. The fact that the resulting surface is saddle-shaped is crucial: there are two states in which the

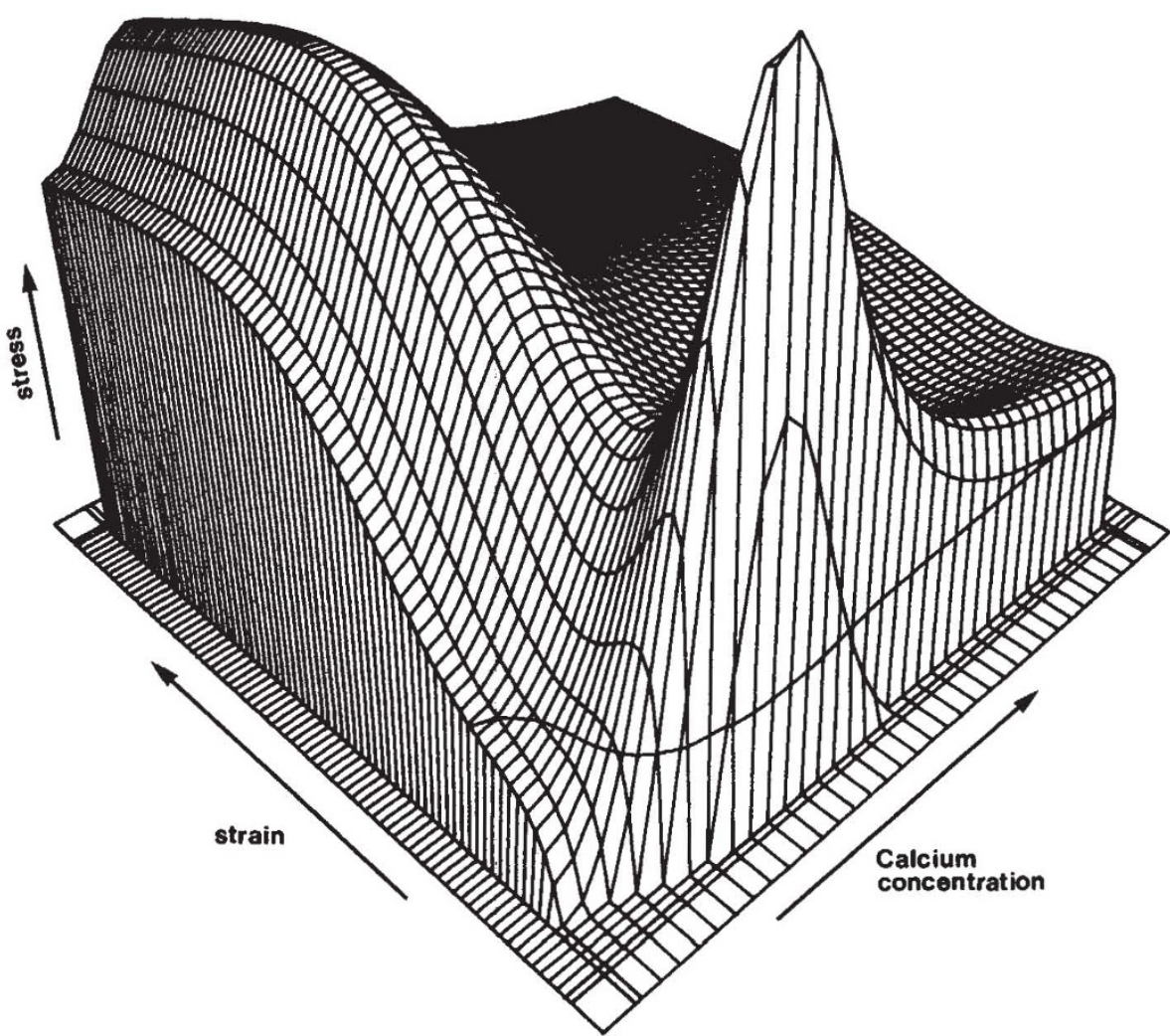

Predicted mechanical properties of an actin-rich cytoplasmic gel. Modified from a paper of G.F. Oster in the Journal of Embryology and Experimental Morphology (ref. 2). gel exerts a large force separated by a region in which it is relatively weak. Consequently, if two separate regions of gel are linked mechanically, one will compete with the other and the combination will be inherently unstable. Built into a physical model of appropriate geometry, this property automatically leads to spontaneous rhythmic mechanochemical oscillations that have a close similarity to those seen in reality.

In a companion paper (which is mathematically less complete), Oster provides a number of valuable insights into the perplexing phenomenon of fibroblast locomotion ${ }^{2}$. He points out, for example, that the fibres in any aqueous gel are under constant tension due to the osmotic tendency of water molecules to disperse them. Partial solation of one region of a gel therefore generates local osmotic pressure and hence local swelling. As a consequence, actin-fragmenting proteins, which solate actin gels in response to calcium signals, could in principle underlie the production of lamellipodia or microspikes from the fibroblast surface.

The potential power of these concepts is illustrated by the wide range of biological phenomena to which they can be applied. One of the earliest models, for example, describes the contractile properties of epithelial cell sheets ${ }^{4}$. In this case, the focus is on the apical actin filament arrays, which are assumed to undergo stretch-induced activation. Thus, when one cell contracts it will stretch other cells in the sheet, causing them to fire in turn, so propagating a wave of invagination. Computations on that basis produce a remarkably life-like simulation of sea urchin gastrulation, formation of the ventral furrow in Drosophila and neural tube formation in amphibia. Other models based on properties of actin gels have given rise to regular two-dimensional patterns of feather primordia and the skeletal rudiments in developing vertebrate limbs 5 .

Odell and Oster readily accept that their mathematical models are unlikely to be true. The equations correctly represent the observations, but the constraints are not too severe. After all, with two or more differential equations to conjure with, one has a hat that can produce any number of rabbits. But, at the least, the models demonstrate the rich potential for coordinated change in the mechanical properties of actin-rich gels. Moreover, they identify crucial questions of gel mechanics that now need to be, and probably can be, answered by experiment.

1. Odell, G.M. J. Emb. exp. Morphol. 83, supp. 261 (1984). 2. Oster, G.F. J. Emb. exp. Morphol 83, supp, 327 (1984). 3. Pollard, T.D. Nature News and Views 312, 403 (1984).

4. Odell, G., Oster, G.F., Burnside, B. \& Alberch, P. Dev. Biol. 85, 446 (1981).

5. Oster, G.F., Murray, J.D. \& Harris, A.K. J. Emb. exp. Morph. 78, 83 (1983).

Dennis Bray is in the Medical Research Council Cell Biophysics Unit, 26-29 Drury Lane, London WC2B SRL, UK. 